\title{
The Qualitative and Quantitative Methods of Kovalevskys Case
}

\author{
Fawzy Mohamed Fahmy El-Sabaa, Alshimaa Abdelbasit Mohamed*, Salma Khalel Zakria \\ Department of Mathematics, Faculty of Education, Ain Shams University, Cairo, Egypt \\ Email: *shaimaaabdelbasset@edu.asu.edu.eg
}

How to cite this paper: El-Sabaa, F.M.F., Mohamed, A.A. and Zakria, S.K. (2017) The Qualitative and Quantitative Methods of Kovalevskys Case. Journal of Applied Mathematics and Physics, 5, 1837-1854. https://doi.org/10.4236/jamp.2017.59155

Received: July 1, 2017

Accepted: September 24, 2017

Published: September 27, 2017

Copyright $\odot 2017$ by authors and Scientific Research Publishing Inc. This work is licensed under the Creative Commons Attribution International License (CC BY 4.0).

http://creativecommons.org/licenses/by/4.0/ c) (i) Open Access

\begin{abstract}
The phase portrait of the functions obtained by Hamilton-Jacobi equations is substantiated, and the classification of singular points is found, and the bifurcation diagram for the problem is studied. The numerical calculation by using Poincaré surface section is used to get the invariant tori for our problem.
\end{abstract}

\section{Keywords}

Hamilton-Jacobi's Equations, Phase Portrait, Poincaré Surface-Section, Qualitative and Quantitative Methods

\section{Introduction}

The problem of the motion of a rigid body with the fixed point in the field of constant gravity is one of the oldest in mechanics [1]. The system of the motion contains three degrees of freedom (Euler's angles). The system possesses three first integrals, and according to Jacobi's last multiplier, the system can be integrated if there are other first integrals. Kovalevskaya [2] proved that the only one-valued first integral can be found in three cases: first, in Euler's case, where the fixed point is located at the center of mass and rotation occurs freely without the influence of torque; second, in the Lagrange's case when the rigid body is a symmetric top with the center of mass on the axis of symmetry; thirdly, in Kovalevskaya's case in which the ellipsoid of inertia about a fixed is symmetric, and the moments of inertia at the supporting point are equal, and each one of them is twice as much as the third one and the center of the mass in the plane of equal moments of inertia. Kovalevskaya [3] showed that other integral cannot be single valued over the whole $t$-complex plane and Hussen [4] found that, when ellipsoid of inertia is not symmetric, a new algebraic integral cannot exist, for arbitrary initial conditions, except in the three cases mentioned above. 
In the case of Kovalevskaya, the problem reduced to quadrature and the integral was obtained through a Remain $\theta$-function of two variables [5]. These functions however are not single-valued, and on the other hand, the functions do not have branch point and hence they are root functions [6]. Consequently, the qualitative and quantitative investigations give us more understanding of the motion of the problem.

\section{The Equations of Motion}

The Euler-Poisson equations for the Kovalevskaya case where two moments of inertia are equal and the third is half of one of them and the center of mass in the equatorial of the ellipsoid of inertia has the form

$$
\begin{aligned}
& 2 \frac{\mathrm{d} p}{\mathrm{~d} t}=q r, \\
& 2 \frac{\mathrm{d} q}{\mathrm{~d} t}=-p r-\gamma_{3}, \\
& \frac{\mathrm{d} r}{\mathrm{~d} t}=\gamma_{2}, \\
& \frac{\mathrm{d} \gamma_{1}}{\mathrm{~d} t}=r \gamma_{2}-q \gamma_{3}, \\
& \frac{\mathrm{d} \gamma_{2}}{\mathrm{~d} t}=p \gamma_{3}-r \gamma_{1}, \\
& \frac{\mathrm{d} \gamma_{1}}{\mathrm{~d} t}=q \gamma_{1}-p \gamma_{2},
\end{aligned}
$$

where $p, q, r, \gamma_{1}, \gamma_{2}, \gamma_{3}$ are the Euler-Poisson variables.

The first integrals are taken the following form and for more details [7].

$$
\begin{gathered}
2\left(p^{2}+q^{2}\right)+r^{2}+2 a \gamma_{1}=3 l_{1}, \\
2\left(p \gamma_{1}+q \gamma_{2}\right)+r \gamma_{3}=l, \\
\gamma_{1}^{2}+\gamma_{2}^{2}+\gamma_{3}^{2}=1, \\
\left(p^{2}-q^{2}-a \gamma_{1}\right)^{2}+2\left(p q-a \gamma_{2}\right)^{2}=k^{2},
\end{gathered}
$$

and the system (1) can be reduced to quadrature

$$
\begin{aligned}
& \frac{\mathrm{d} s_{1}}{\sqrt{\Phi\left(s_{1}\right)}}+\frac{\mathrm{d} s_{2}}{\sqrt{\Phi\left(s_{2}\right)}}=0, \\
& \frac{s_{1} \mathrm{~d} s_{1}}{\sqrt{\Phi\left(s_{1}\right)}}+\frac{s_{2} \mathrm{~d} s_{2}}{\sqrt{\Phi\left(s_{2}\right)}}=\frac{\mathrm{d} t}{2},
\end{aligned}
$$

where the Kovalevskaya polynomial $\Phi(s)$ is defined as

$$
\Phi(s)=\left[s\left[\left(s-6 l_{1}\right)^{2}+1-k^{2}\right]-2 l^{2}\right]\left[\left(s-3 l_{1}-k\right)\left(s-3 l_{1}+k\right)\right] .
$$

Kolossoff [8] introduced the potential

$$
V=\frac{x^{2}+y^{2}-k x+1}{\sqrt{x^{2}+y^{2}}},
$$

and with the new time $\tau$ through the relation 


$$
i \mathrm{~d} \tau=\frac{2\left(r x_{1}+\gamma_{3}\right)\left(r x_{2}+\gamma_{3}\right)}{\left(x_{1}-x_{2}\right)^{2}} \mathrm{~d} t,
$$

and then system (1) can be converted to the plan motion of massless point defined as

$$
x^{\prime \prime}=\frac{\partial V}{\partial x}, \quad y^{\prime \prime}=\frac{\partial V}{\partial y}, \quad\left('=\frac{\mathrm{d}}{\mathrm{d} \tau}\right)
$$

and possess the energy integral

$$
\frac{1}{2}\left(\dot{x}^{2}+\dot{y}^{2}\right)+V=h
$$

under the condition that the constants of energy of both systems are equal

$$
3 l_{1}=h,
$$

Introducing the elliptic coordinates $\lambda, \mu$ such that

$$
\begin{aligned}
& x=\frac{\lambda \mu}{k}+k, \\
& y=\frac{1}{k} \sqrt{\left[\left(\lambda^{2}-k^{2}\right)\left(k^{2}-\mu^{2}\right)\right]},
\end{aligned}
$$

with Jacobian matrix $M$ defined as

$$
M=\left(\begin{array}{ll}
\frac{\partial x}{\partial \lambda} & \frac{\partial x}{\partial \mu} \\
\frac{\partial y}{\partial \lambda} & \frac{\partial y}{\partial \mu}
\end{array}\right),
$$

one can give the momenta $p_{x}, p_{y}$ as function of $p_{\lambda}, p_{\mu}$, such that

$$
\left(\begin{array}{l}
p_{x} \\
p_{y}
\end{array}\right)=M^{-1}\left(\begin{array}{l}
p_{\lambda} \\
p_{\mu}
\end{array}\right) .
$$

Then the canonical variables $\left(p_{\lambda}, p_{\mu}, \lambda, \mu\right)$ are given by

$$
\begin{aligned}
& p_{x}=\frac{\left(\lambda^{2}-k^{2}\right) \mu p_{\lambda}-\left(\mu^{2}-k^{2}\right) \lambda p_{\mu}}{k\left(\lambda^{2}-\mu^{2}\right)}, \\
& p_{y}=\frac{\sqrt{\left[\left(\lambda^{2}-k^{2}\right)\left(k^{2}-\mu^{2}\right)\right]}\left(\lambda p_{\lambda}-\mu p_{\mu}\right)}{k\left(\lambda^{2}-\mu^{2}\right)} .
\end{aligned}
$$

The system is a Liouville system, where the constants of integration are founded from

$$
\begin{aligned}
& \left(\lambda^{2}-k^{2}\right) p_{\lambda}^{2}+2 \lambda^{3}+2\left(1-k^{2}\right) \lambda-2 \lambda^{2} h \\
& =\left(\mu^{2}-k^{2}\right) p_{\mu}^{2}+2 \mu^{3}+2\left(1-k^{2}\right) \mu-2 \mu^{2} h=C,
\end{aligned}
$$

and the momenta $p_{\lambda}$ and $p_{\mu}$ are given by

$$
p_{\lambda}=\sqrt{\frac{\Phi(\lambda)}{\lambda^{2}-k^{2}}},
$$




$$
p_{\mu}=\sqrt{\frac{\Phi(\mu)}{\mu^{2}-k^{2}}} .
$$

By using Hamilton-Jacob method, we obtain

$$
\begin{aligned}
& \frac{\mathrm{d} \lambda}{\sqrt{\phi(\lambda)\left(\lambda^{2}-k^{2}\right)}}+\frac{\mathrm{d} \mu}{\sqrt{\phi(\mu)\left(\mu^{2}-k^{2}\right)}}=0, \\
& \frac{\lambda \mathrm{d} \lambda}{\sqrt{\phi(\lambda)\left(\lambda^{2}-k^{2}\right)}}+\frac{\mu \mathrm{d} \mu}{\sqrt{\phi(\mu)\left(\mu^{2}-k^{2}\right)}}=\frac{\mathrm{d} \tau}{\sqrt{2}(\lambda+\mu)},
\end{aligned}
$$

and with time changing

$$
\frac{\mathrm{d} \tau}{\sqrt{2}(\lambda+\mu)}=\mathrm{d} t
$$

the Equation (16) are the same as the Kovalevskaya equations, where the polynomial

$$
p(u)=\left(u^{2}-k^{2}\right) \phi(u),
$$

corresponds to the Kovalevskaya polynomial $\Phi(s)$.

Accordingly the problem of the integration of Kovalevskaya top is equivalent to the problem of the plane motion of the massless point, and then we study the problem in Kolossoff's variable $\left(p_{\lambda}, p_{\mu}, \lambda, \mu\right)$.

\section{Phase Portrait of the Separated Functions}

Consider the function

$$
f=q^{3}+q\left(1-k^{2}\right)+h\left(q^{2}+1-k^{2}\right)-p^{2}\left(k^{2}-q^{2}\right) .
$$

We construct the lines of constant $f$ on the plane $(p, q)$, which is called the phase portrait of $f$. The phase portrait helps us to find the topological interpretation of the trajectory as follows: if the roots of the function are distinct for given initial values of $(p, q)$, then $P\left(p_{1}, p_{2}\right)$ and $Q\left(q_{1}, q_{2}\right)$ will change in a periodic manner, but if the function has multiple roots, then we have an infinity of motion, which gives an asymptotic solution of the canonical equations. Thus the study of lines of constant $f$ provides a complete picture of the bifurcations of our problem.

We first study the singular points and it is distinguished by using Gaussian curvature of $f$. These points can be found from the equations

$$
\begin{aligned}
& \frac{\partial f}{\partial p}=-p\left(k^{2}-q^{2}\right)=0, \\
& \frac{\partial f}{\partial q}=3 q^{2}+2 h q+1-k^{2}+2 p^{2} q=0,
\end{aligned}
$$

and hence we have the following: where $p=0$, we get

$$
3 q^{2}+2 h q+1-k^{2}=0,
$$

and when $q= \pm k$, we get the two equations 


$$
\begin{aligned}
& p^{2}=-h-k-\frac{1}{2 k}, \\
& p^{2}=-h+k+\frac{1}{2 k},
\end{aligned}
$$

The discriminant of $(21)$ is

$$
f_{1}=h^{2}+3 k^{2}-3
$$

and from (22) the two functions are $f_{2}, f_{3}$

$$
\begin{aligned}
& f_{2}=-2 h k-2 k^{2}-1, \\
& f_{3}=-2 h k+2 k^{2}+1 .
\end{aligned}
$$

It is clear that the curve $f_{1}=0$ is tangent to the branches of the curves $f_{2}$ and $f_{3}$ at the points $k= \pm \frac{1}{2}$. This can be found from the consideration that the equation $f_{1}=f_{2}$ gives the two roots $\left(k_{1}, h_{1}\right),\left(k_{2}, h_{2}\right)$ such that sign $k_{1} \cdot \operatorname{sign} k_{2}<0$.

From $f_{2}=0$ and $f_{1}=0$, we get

$$
\begin{gathered}
h=-k-\frac{1}{2 k} \\
4 k^{2}+\frac{1}{4 k^{2}}-2=0
\end{gathered}
$$

Now, we study the points in the domain $D_{i}$ where $i=1,2, \cdots, 16$, Figure 1 .

The regions Di of real motion on the $(h, k)$ plane.

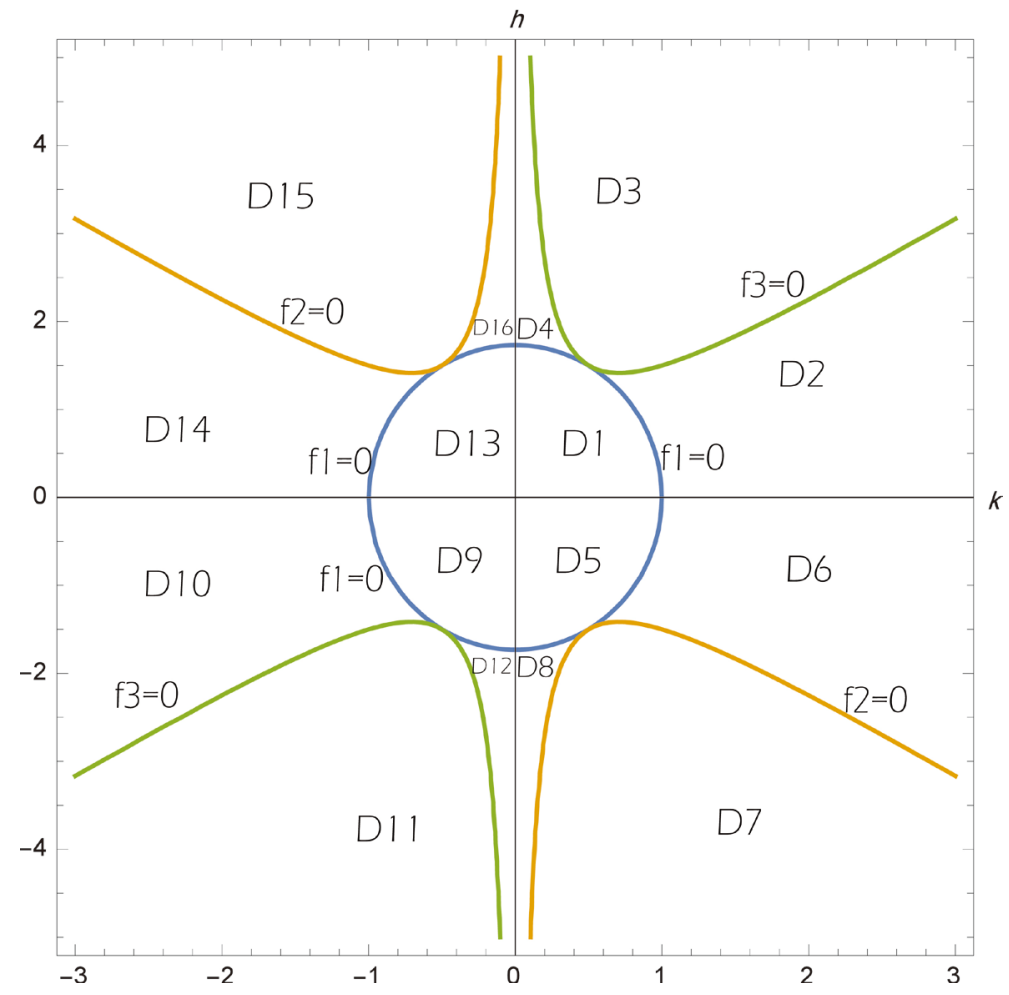

Figure 1. The regions $D_{i}$ of real motions on the $(k, h)$ plane. 
1) The Domain $D_{1}: f_{1}<0, f_{2}<0, f_{3}>0$

Equations ((21) and (22)) are not solved when $p=0, q=k$ and then there are no singular points of $f$ on the line $p=0$ and $q=k$, but when $q=-k$, there are two singular points with $p$ coordinates,

$$
p= \pm\left(\frac{2 k^{2}-2 h k+1}{2 k}\right)^{\frac{1}{2}} \text {. }
$$

To get these types of points, put

$$
\begin{aligned}
& q=-k+y, \\
& p= \pm\left(\frac{2 k^{2}-2 h k+1}{2 k}\right)^{\frac{1}{2}}+x,
\end{aligned}
$$

in the function $f$, neglecting terms of degree greater than 2 , then we have

$$
f=\frac{1-4 k^{2}}{2 k} y^{2} \mp 4 k\left(\frac{2 k^{2}-2 h k+1}{2 k}\right)^{\frac{1}{2}} x y+A_{0},
$$

where $A_{0}$ contains the terms of zeros and first degree of $x, y$.

The singular points are hyperbolic points, where

$$
\left[\begin{array}{ll}
\frac{\partial^{2} f}{\partial x^{2}} & \frac{\partial^{2} f}{\partial x \partial y} \\
\frac{\partial^{2} f}{\partial x \partial y} & \frac{\partial^{2} f}{\partial y^{2}}
\end{array}\right]_{x=y=0}<0
$$

and when $p=0$, we have the two singular points: $\left(\frac{-h \pm \sqrt{h^{2}+3\left(k^{2}-1\right)}}{3}, 0\right)$.

In the same manner we can get the type of points in the domains $D_{i}(i=1, \cdots, 16)$, they are shown in Figures 2-20, and summarize that in Table 1.

\section{The Integration of the Problem}

To complete the picture of our problem, we solve the kolossoff system numerically by using a surface section introducing by Poincaré [6].

A surface of section is a way of presenting a trajectory in $\mathrm{n}$-dimension phase space in an (n-1) dimension space. By picking one phase element constant and plotting the value of the other element each time the selected element has the desired value, an intersection surface is obtained. Accordingally the solution of system (7) represents itself the trajectory in the phase space $(x, \grave{x}, y, \grave{y})$, and on the energy surface $h=$ constant, the trajectory may be treated in the three dimensional invariant hypersurface $(x, \grave{x}, y)$. The successive points with $\grave{y}>0$ in at which an orbit crosses the plane $x=0$ in phase space represent a locus of two-dimensional area-preserving mapping on the $(x, \grave{x})$ plane, which is called Poincaré mapping. 


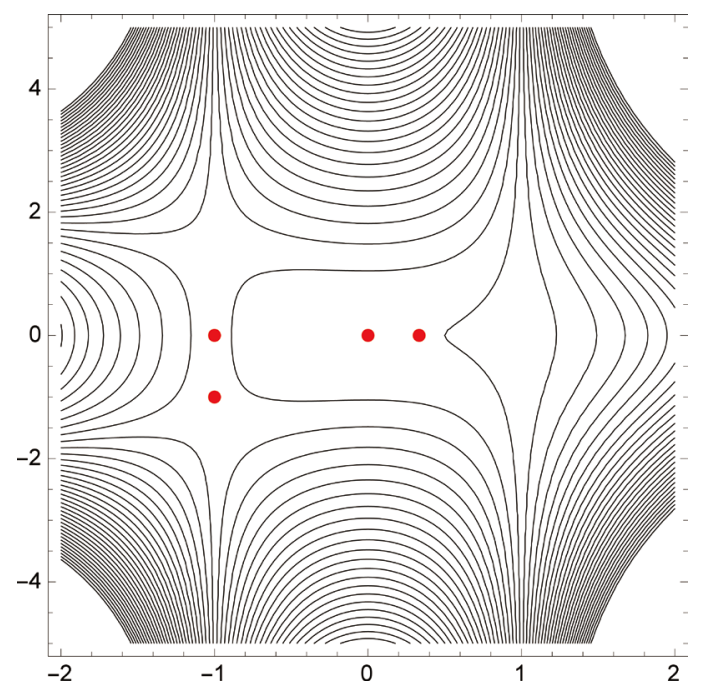

Figure 2. The four-hyperbolic points in domain $D_{1}$.

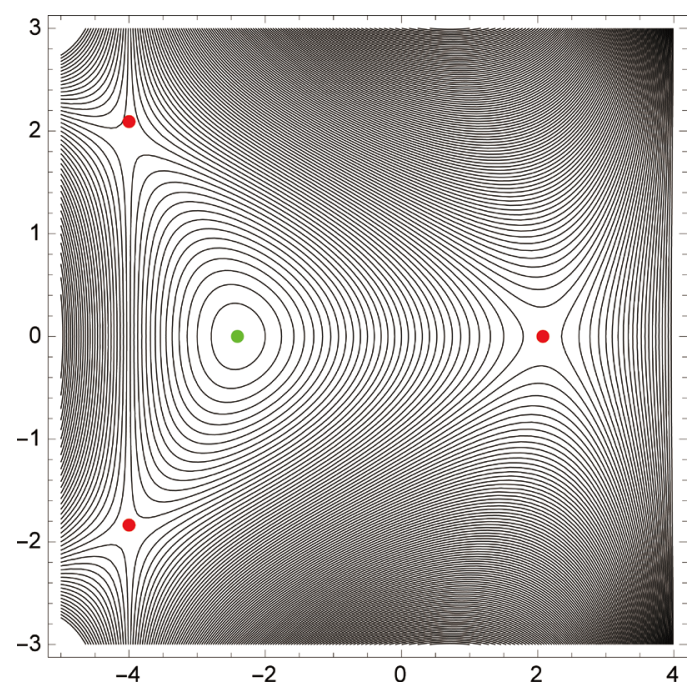

Figure 3. The Three-hyperbolic points and one-elliptic point in domain $D_{2}$.

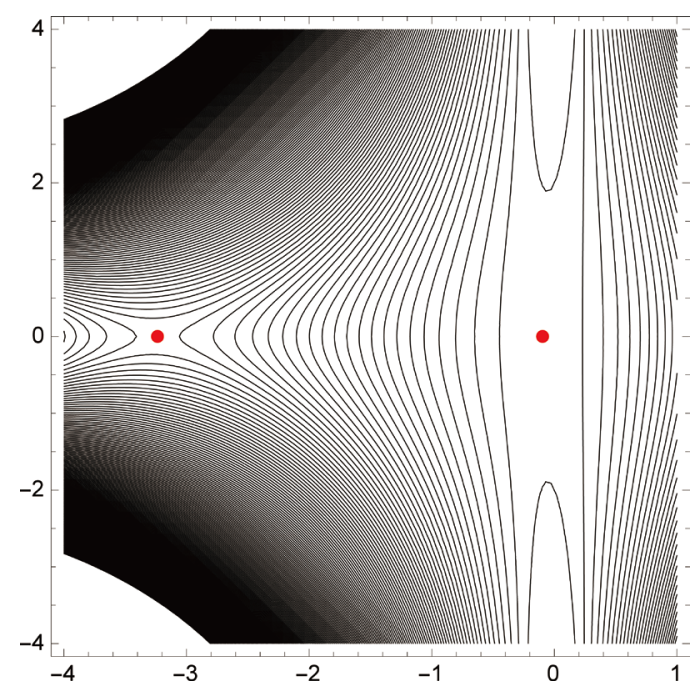

Figure 4. The two-hyperbolic points in domain $D_{3}$. 


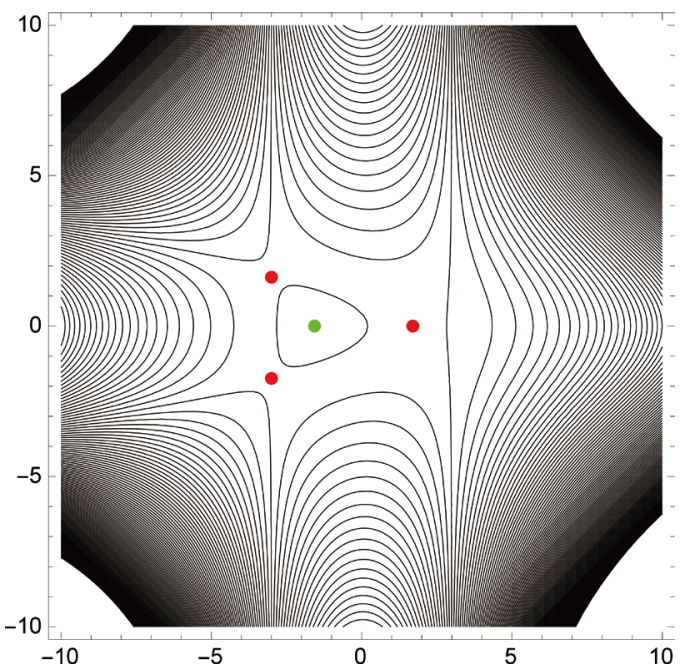

Figure 5. The three-hyperbolic points and one elliptic point in domain $D_{4}$.

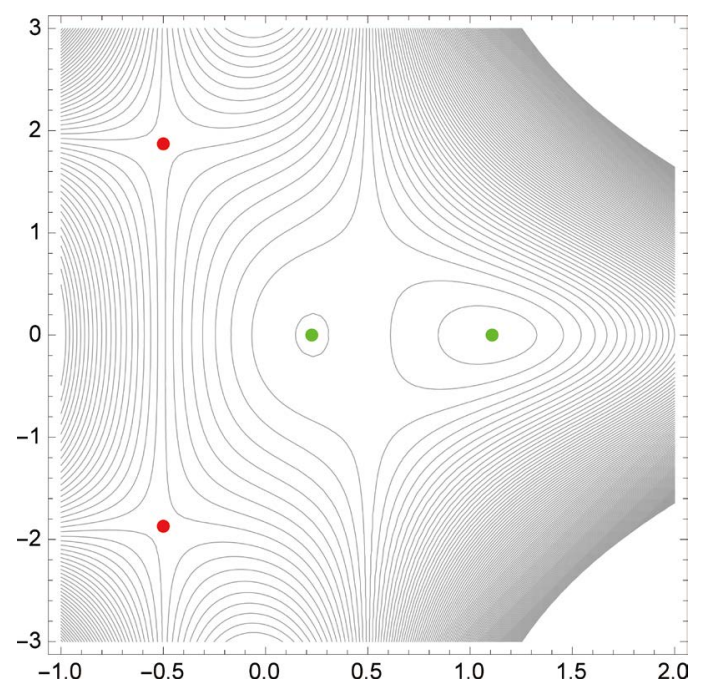

Figure 6. The two-elliptic points and two-hyperbolic points in domain $D_{5}$.

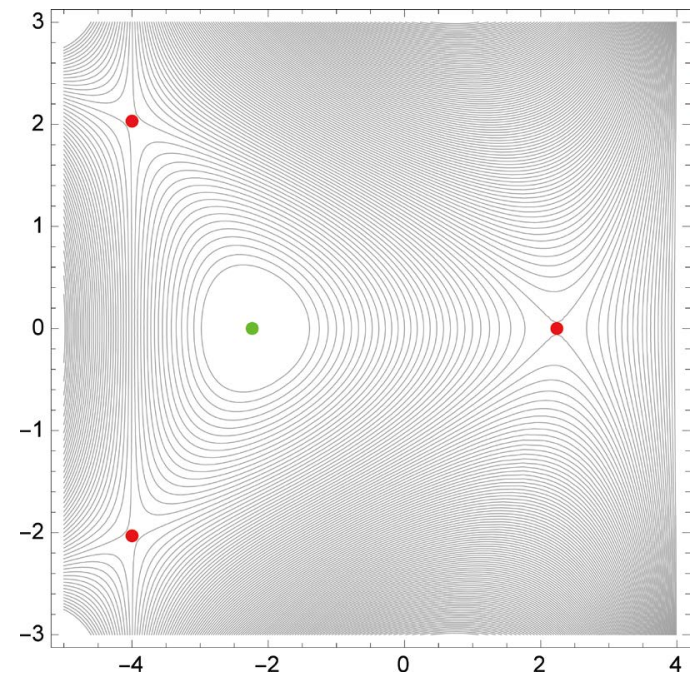

Figure 7. The three-hyperbolic points and one-elliptic point in domain $D_{6}$. 


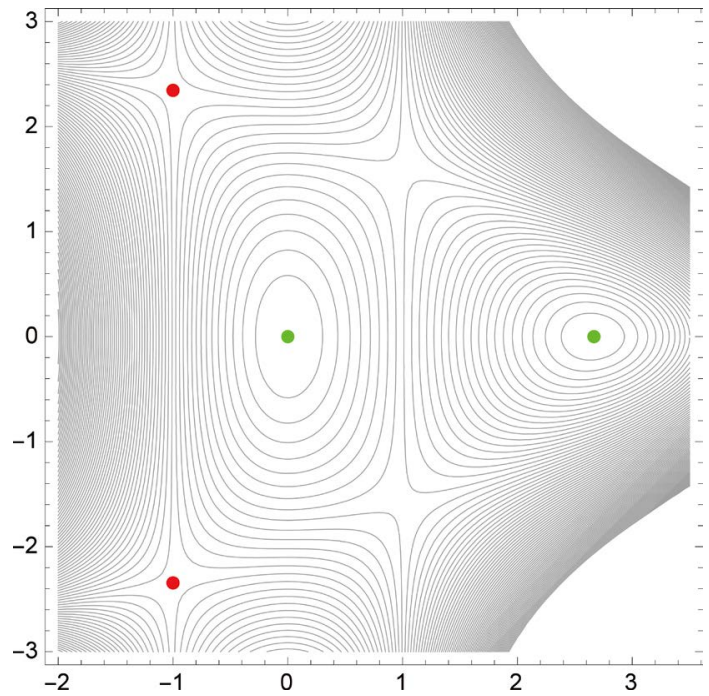

Figure 8. The two-hyperbolic points and two-elliptic points in domain $D_{7}$.

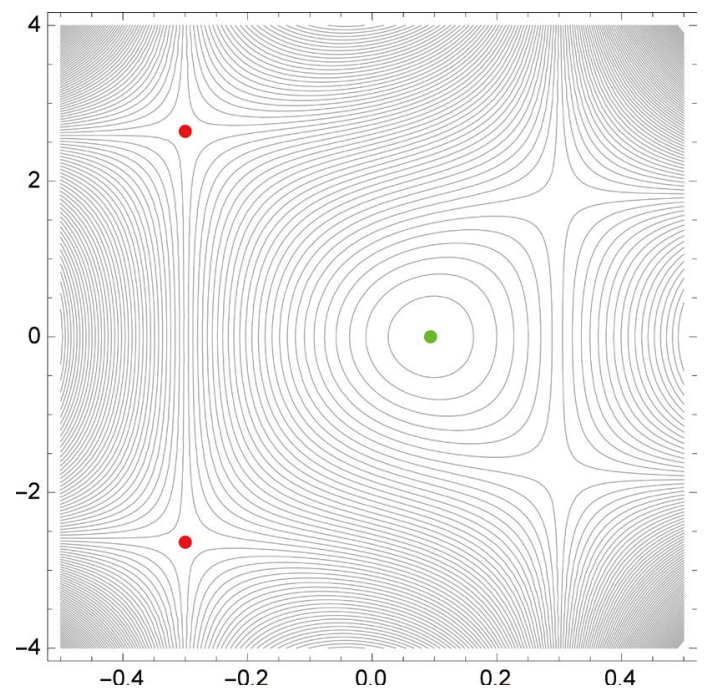

Figure 9. The two-hyperbolic points and one-elliptic point in domain $D_{8}$.

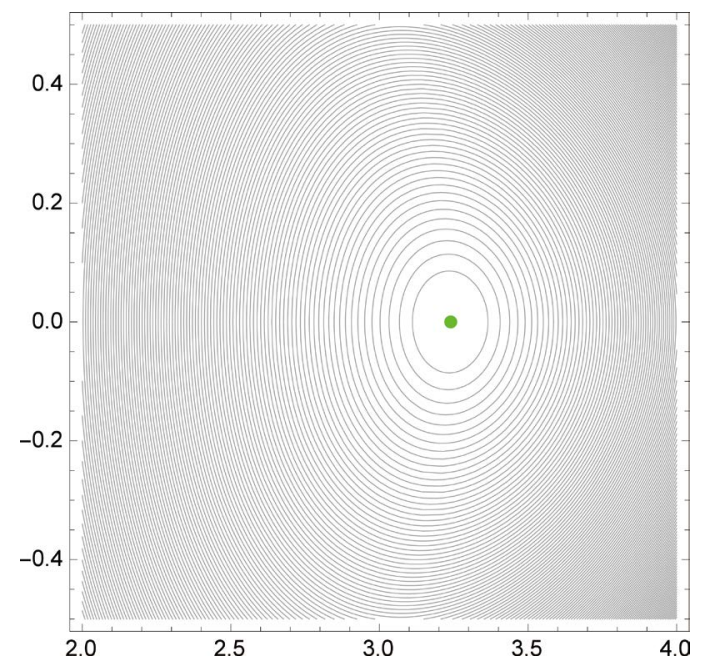

Figure 10. The one-elliptic point in domain $D_{8}$. 


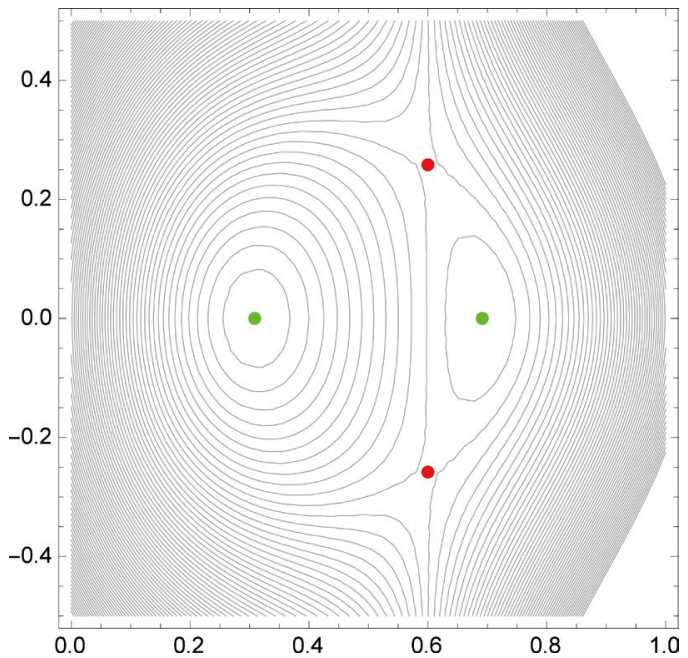

Figure 11. The two-hyperbolic points and two-elliptic points in domain $D_{9}$.

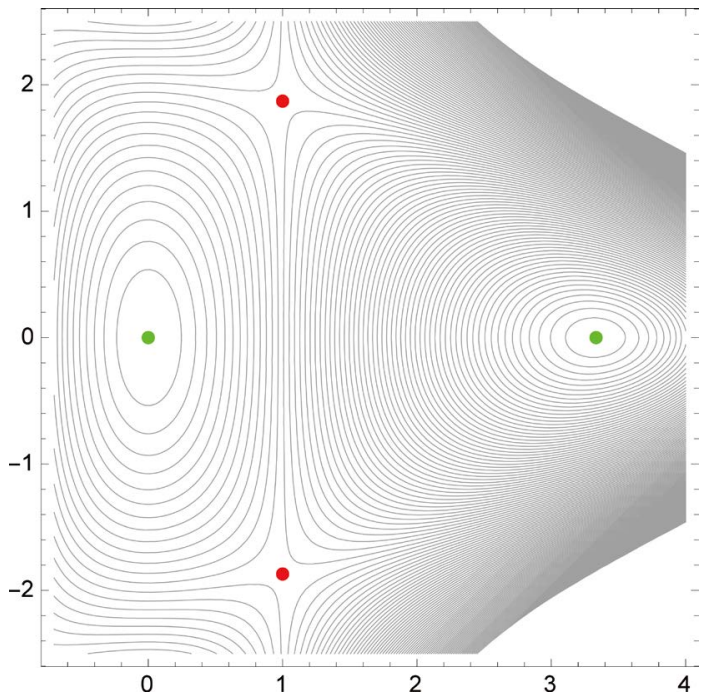

Figure 12. The one-hyperbolic point and one-elliptic point in domain $D_{10}$.

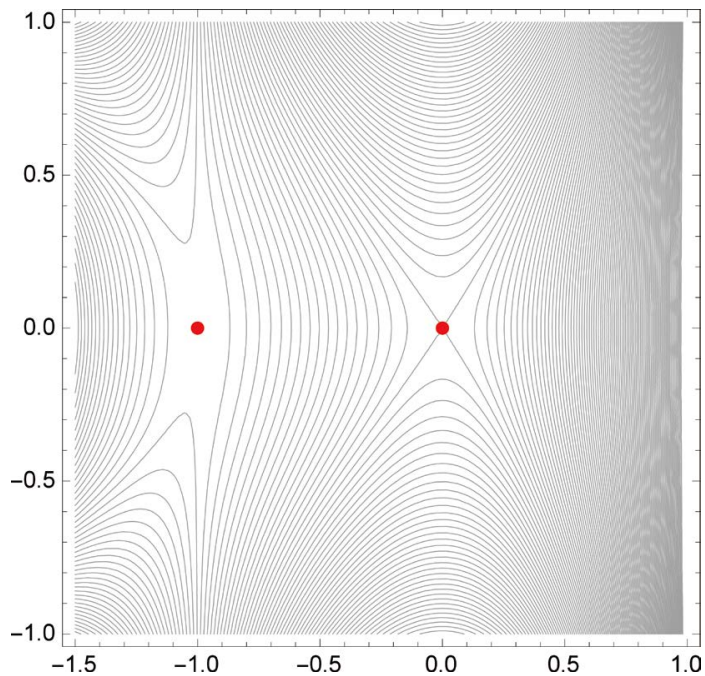

Figure 13. The two-elliptic points and two-hyperbolic points in domain $D_{11}$. 


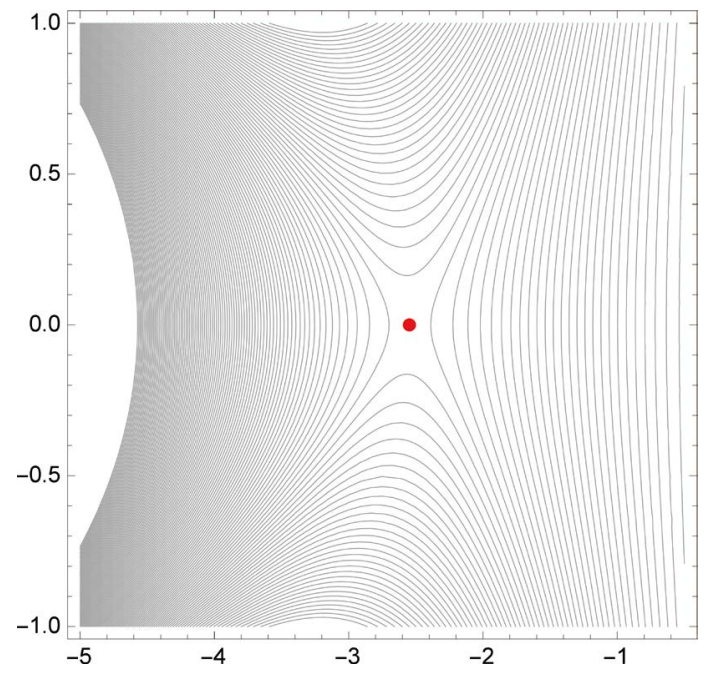

Figure 14. The two-hyperbolic points in domain $D_{12}$.

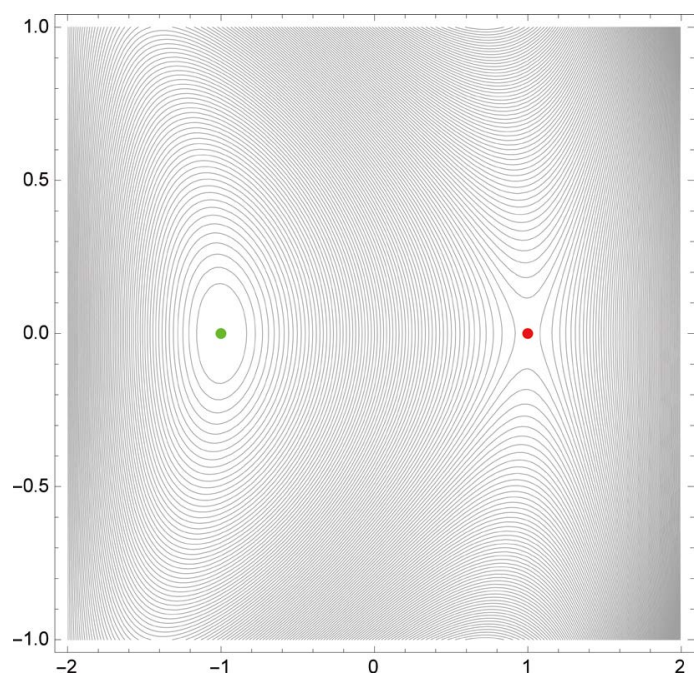

Figure 15. The two-elliptic points in domain $D_{12}$.

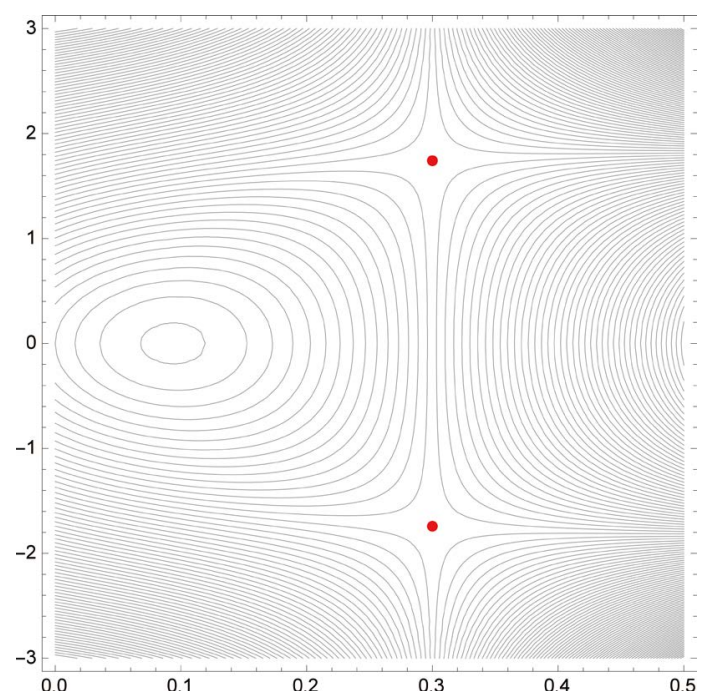

Figure 16. The two-hyperbolic points in domain $D_{13}$. 


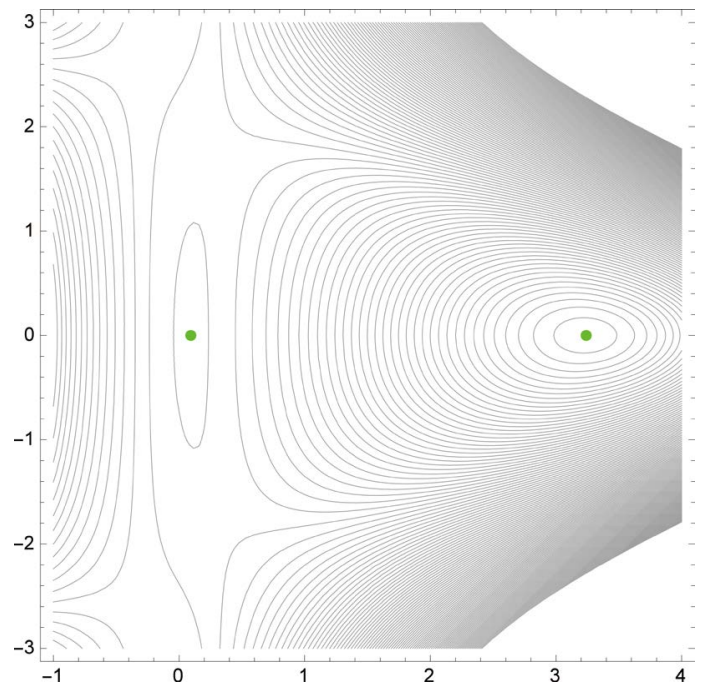

Figure 17. The one-hyperbolic point and one-elliptic point in domain $D_{14}$.

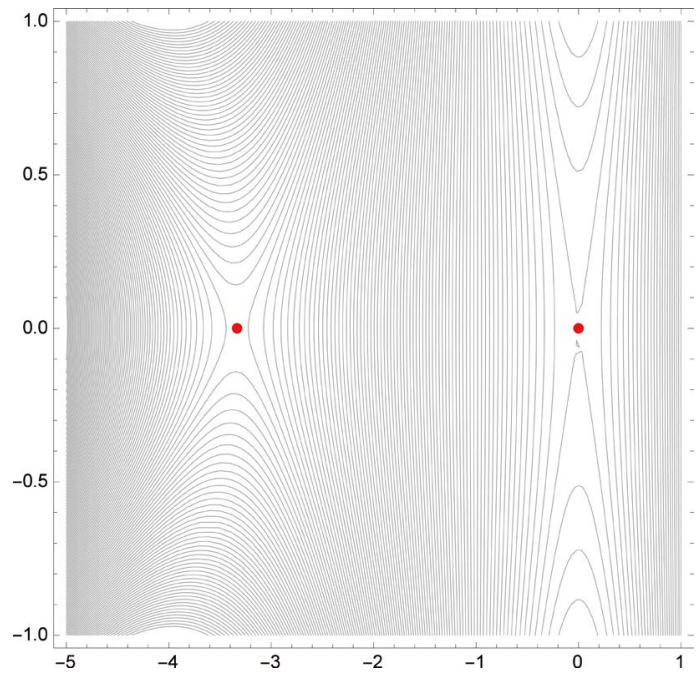

Figure 18. The two-hyperbolic points in domain $D_{15}$.

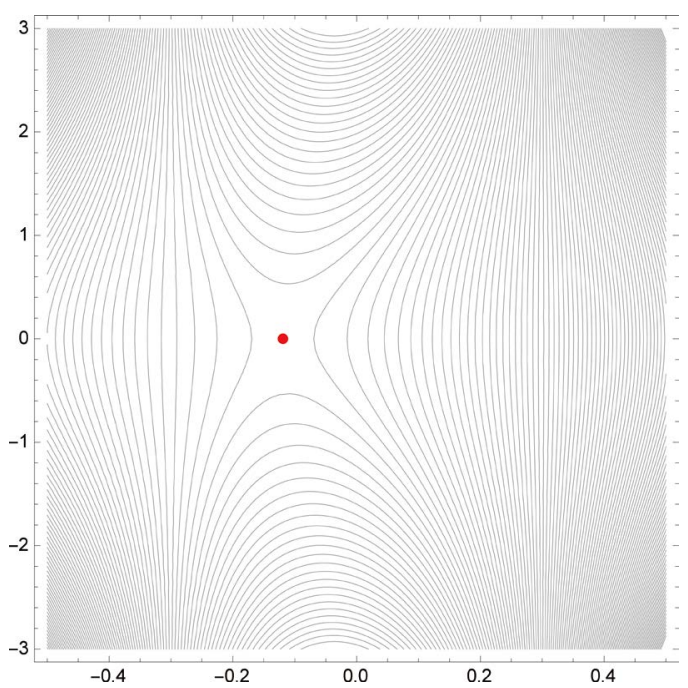

Figure 19. The one-hyperbolic point in domain $D_{16}$. 


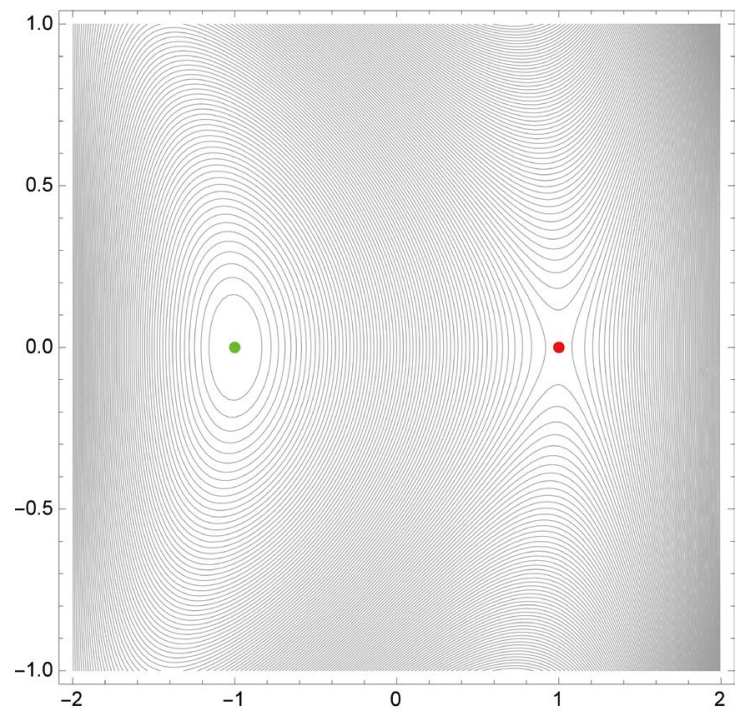

Figure 20. The one-hyperbolic point in domain $D_{16}$.

If we put $\grave{x}=\grave{y}=0$ in (8), then we have the equipotential lines

$$
V(x, y)=h \text {. }
$$

Figure 21 shows the equipotential lines for different values of $h$ and $k$. Putting $\grave{x}=0$ in integral energy equation, we have

$$
\grave{y}=\sqrt{2(h-V(0, y))}
$$

where the curve of zero velocity is

$$
h-V(0, y)=0 .
$$

Figure 22 shows the curve of zero velocity, its clear that the curves is independent of Kovalevsky constant $k$.

Now the initial values of $\tau=\tau_{0}$ is $\left(x=\grave{x}=0, y_{0}, \grave{y}_{0}\right)$ and then we get the point $p_{1}\left(y_{1}, \grave{y}_{1}\right)$ at $\tau=\tau_{1}$. The point $p_{0}$ is the mapped into $p_{1}$, or $T_{p_{0}} \rightarrow p_{1}$, so if we plot the successive intersections of the motion with the surface of section, they will in general occur anywhere within a bounded area of the plane and must lie on a unique curve.

Hénon-Heiles $H-H$ [9] are used the Poincar $e^{\prime}$ section-surface to prove that they exist, the third integral of a non-linear motion of star around a galactic center with the motion restricted to plane, for some values of the constant of energy, the motion is ordered, and with increasing the energy, the ordered motion becomes stochastic. The $H-H$ model [10] becomes a criterion for the existence of the integral of any conservative system. By using these criteria in our problem, we find that, the image points of the initial points, lie on invariant curve. Therefore, these orbits lie on invariant tori and the motion is ordered. Figures 23-28 show the invariant curves for different values of $k$ and $h$ and they are symmetric with respect to $y$ and $\grave{y}$. 
Table 1. The type of points in the domains $D_{i}$.

\begin{tabular}{|c|c|c|c|}
\hline Domain & The points & Types of points & Figures \\
\hline$D_{1}: f_{1}<0, f_{2}<0, f_{3}>0$ & $\left(\frac{-h \pm \sqrt{h^{2}+3\left(k^{2}-1\right)}}{3}, 0\right),\left(-k, \pm \sqrt{\frac{2 k^{2}-2 h k+1}{2 k}}\right)$ & Four-hyperbolic points & Figure 2 \\
\hline$D_{2}: f_{1}>0, f_{2}<0, f_{3}>0$ & $\left.\frac{-h \pm \sqrt{h^{2}+3\left(k^{2}-1\right)}}{3}, 0\right),\left(-k, \pm \sqrt{\frac{2 k^{2}-2 h k+1}{2 k}}\right.$ & $\begin{array}{l}\text { Three-hyperbolic points and one elliptic } \\
\text { point }\end{array}$ & Figure 3 \\
\hline$D_{3}: f_{1}>0, f_{2}<0, f_{3}<0$ & $\left(\frac{-h \pm \sqrt{h^{2}+3\left(k^{2}-1\right)}}{3}, 0\right)$ & Two-hyperbolic points & Figure 4 \\
\hline$D_{4}: f_{1}>0, f_{2}<0, f_{3}>0$ & $\left(\frac{-h \pm \sqrt{h^{2}+3\left(k^{2}-1\right)}}{3}, 0\right),\left(-k, \pm \sqrt{\frac{2 k^{2}-2 h k+1}{2 k}}\right)$ & $\begin{array}{l}\text { Three-hyperbolic points and one elliptic } \\
\text { point }\end{array}$ & Figure 5 \\
\hline$D_{5}: f_{1}<0, f_{2}<0, f_{3}>0$ & $\left(\frac{-h \pm \sqrt{h^{2}+3\left(k^{2}-1\right)}}{3}, 0\right),\left(-k, \pm \sqrt{\frac{2 k^{2}-2 h k+1}{2 k}}\right)$ & $\begin{array}{l}\text { Two-hyperbolic points and Two-elliptic } \\
\text { points }\end{array}$ & Figure 6 \\
\hline$D_{6}: f_{1}>0, f_{2}<0, f_{3}>0$ & $\left(\frac{-h \pm \sqrt{h^{2}+3\left(k^{2}-1\right)}}{3}, 0\right),\left(-k, \pm \sqrt{\frac{2 k^{2}-2 h k+1}{2 k}}\right)$ & $\begin{array}{l}\text { Three-hyperbolic points and one elliptic } \\
\text { point }\end{array}$ & Figure 7 \\
\hline$D_{7}: f_{1}>0, f_{2}>0, f_{3}<0$ & $\left(\frac{-h \pm \sqrt{h^{2}+3\left(k^{2}-1\right)}}{3}, 0\right),\left(-k, \pm \sqrt{\frac{2 k^{2}-2 h k+1}{2 k}}\right)$ & $\begin{array}{l}\text { Two-hyperbolic points and two-elliptic } \\
\text { points }\end{array}$ & Figure 8 \\
\hline$D_{8}: f_{1}>0, f_{2}>0, f_{3}<0$ & $\left(\frac{-h \pm \sqrt{h^{2}+3\left(k^{2}-1\right)}}{3}, 0\right),\left(-k, \pm \sqrt{\frac{2 k^{2}-2 h k+1}{2 k}}\right)$ & $\begin{array}{l}\text { Two-hyperbolic points and two-elliptic } \\
\text { points }\end{array}$ & $\begin{array}{l}\text { Figure 9, } \\
\text { Figure } 10\end{array}$ \\
\hline$D_{9}: f_{1}<0, f_{2}<0, f_{3}>0$ & $\left(\frac{-h \pm \sqrt{h^{2}+3\left(k^{2}-1\right)}}{3}, 0\right),\left(-k, \pm \sqrt{\frac{2 k^{2}-2 h k+1}{2 k}}\right)$ & $\begin{array}{l}\text { Two-hyperbolic points and two-elliptic } \\
\text { points }\end{array}$ & Figure 11 \\
\hline$D_{10}: f_{1}<0, f_{2}<0, f_{3}>0$ & $\left(\frac{-h \pm \sqrt{h^{2}+3\left(k^{2}-1\right)}}{3}, 0\right)$ & $\begin{array}{l}\text { One-hyperbolic point and one-elliptic } \\
\text { point }\end{array}$ & Figure 12 \\
\hline$D_{11}: f_{1}>0, f_{2}>0, f_{3}<0$ & $\left(\frac{-h \pm \sqrt{h^{2}+3\left(k^{2}-1\right)}}{3}, 0\right),\left(-k, \pm \sqrt{\frac{2 k^{2}-2 h k+1}{2 k}}\right)$ & $\begin{array}{l}\text { Two-hyperbolic points and two-elliptic } \\
\text { points }\end{array}$ & Figure 13 \\
\hline$D_{12}: f_{1}>0, f_{2}<0, f_{3}>0$ & $\left(\frac{-h \pm \sqrt{h^{2}+3\left(k^{2}-1\right)}}{3}, 0\right),\left(-k, \pm \sqrt{\frac{2 k^{2}-2 h k+1}{2 k}}\right)$ & $\begin{array}{l}\text { Two-hyperbolic points and two-elliptic } \\
\text { points }\end{array}$ & $\begin{array}{l}\text { Figure 14, } \\
\text { Figure } 15\end{array}$ \\
\hline$D_{13}: f_{1}<0, f_{2}<0, f_{3}>0$ & $\left(k, \pm \sqrt{\frac{-2 k^{2}-2 h k-1}{2 k}}\right)$ & Two-hyperbolic points & Figure 16 \\
\hline$D_{14}: f_{1}>0, f_{2}<0, f_{3}>0$ & $\left(\frac{-h \pm \sqrt{h^{2}+3\left(k^{2}-1\right)}}{3}, 0\right)$ & One-hyperbolic point and one elliptic point & Figure 17 \\
\hline$D_{15}: f_{1}>0, f_{2}<0, f_{3}<0$ & $\left(\frac{-h \pm \sqrt{h^{2}+3\left(k^{2}-1\right)}}{3}, 0\right)$ & Two-hyperbolic points & Figure 18 \\
\hline$D_{16}: f_{1}>0, f_{2}<0, f_{3}>0$ & $\left(\frac{-h \pm \sqrt{h^{2}+3\left(k^{2}-1\right)}}{3}, 0\right)$ & Two-hyperbolic points & $\begin{array}{l}\text { Figure 19, } \\
\text { Figure } 20\end{array}$ \\
\hline
\end{tabular}




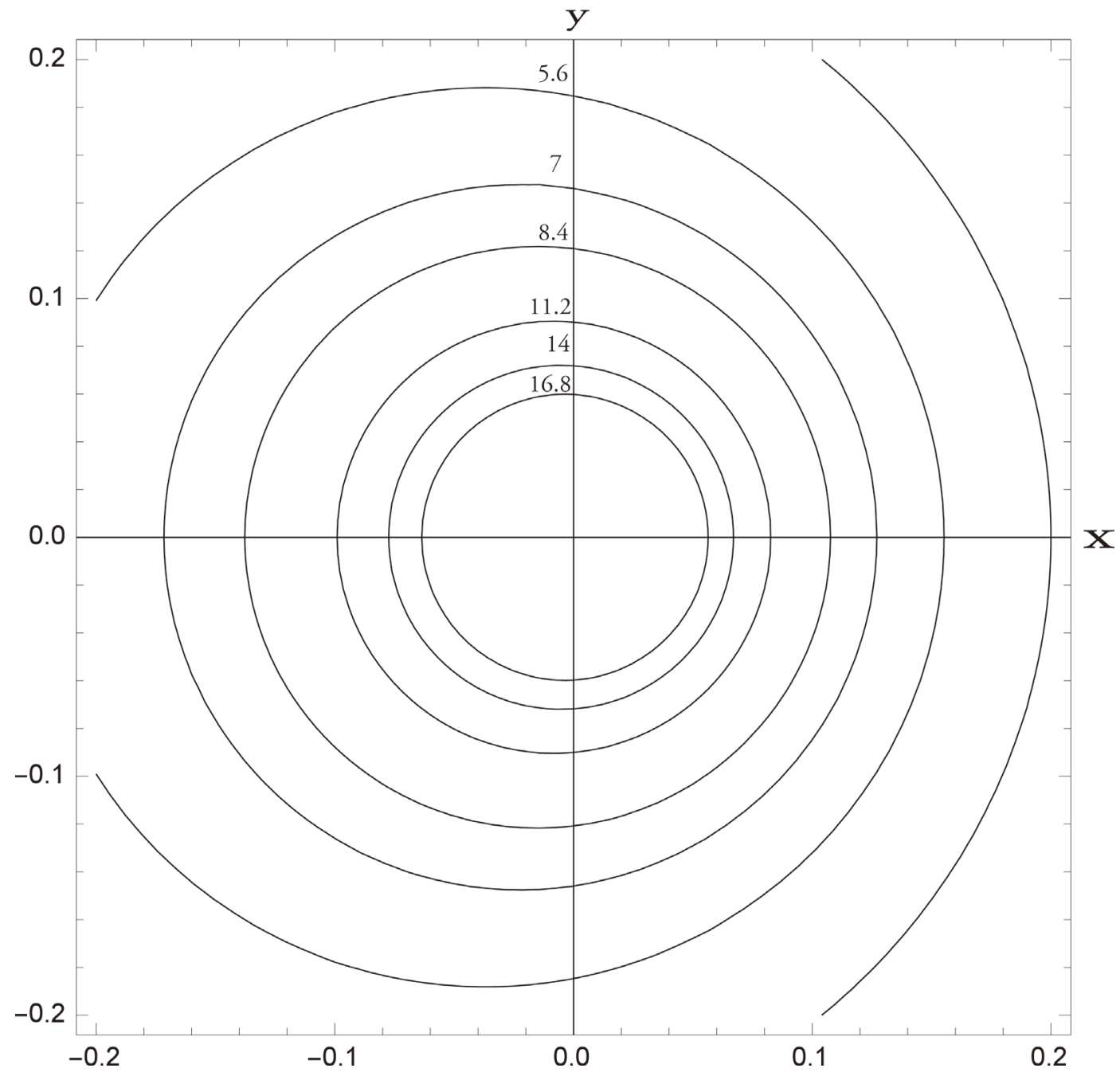

Figure 21. The equipotential line with a different value of $h$.

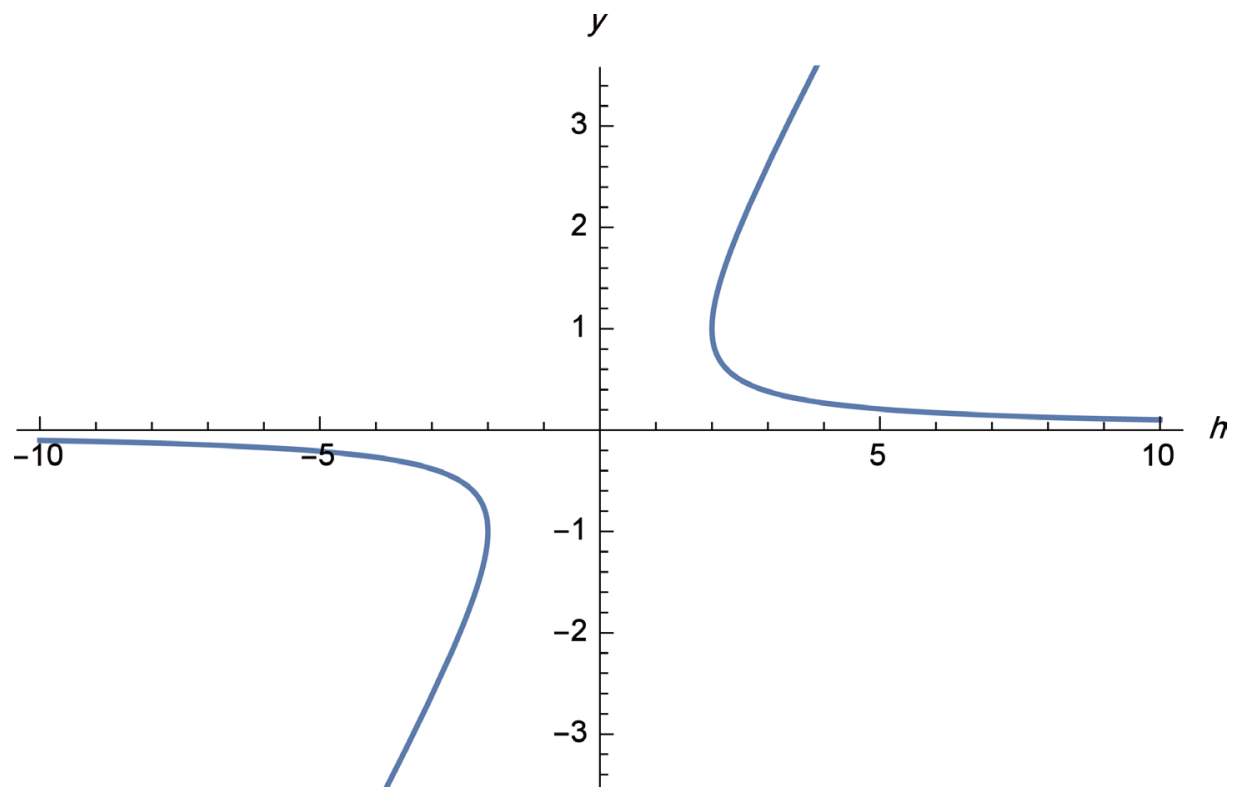

Figure 22. The curve of zero velocity, where $-2 \geq h \geq 2$. 


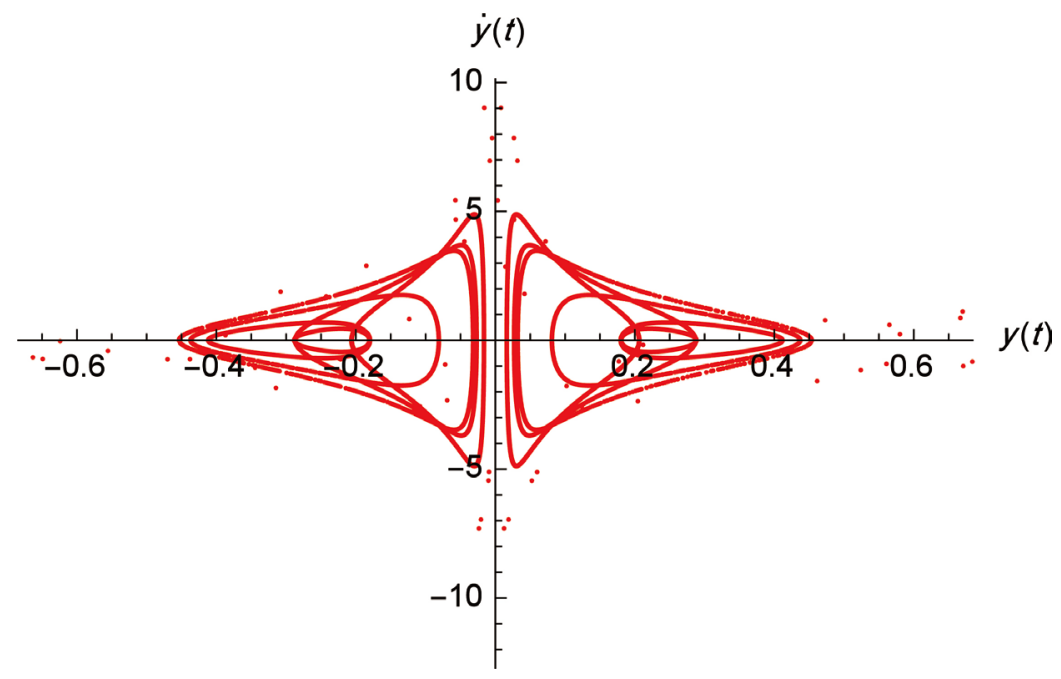

Figure 23. The invariant curves for $h=k=0$.

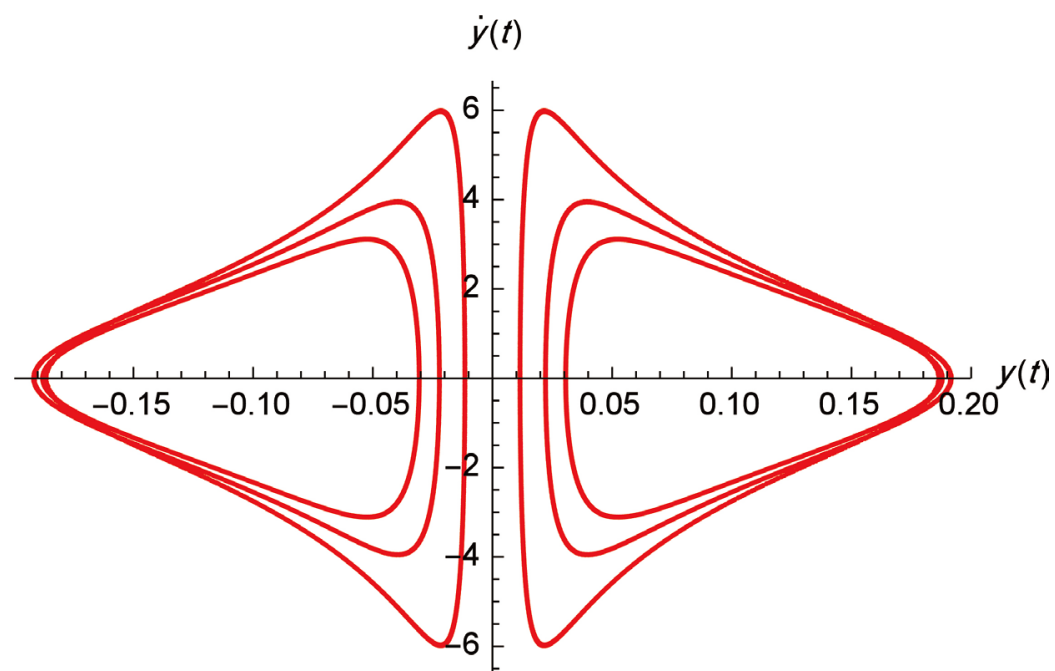

Figure 24. The invariant curves for $h<0$ and $k=0$.

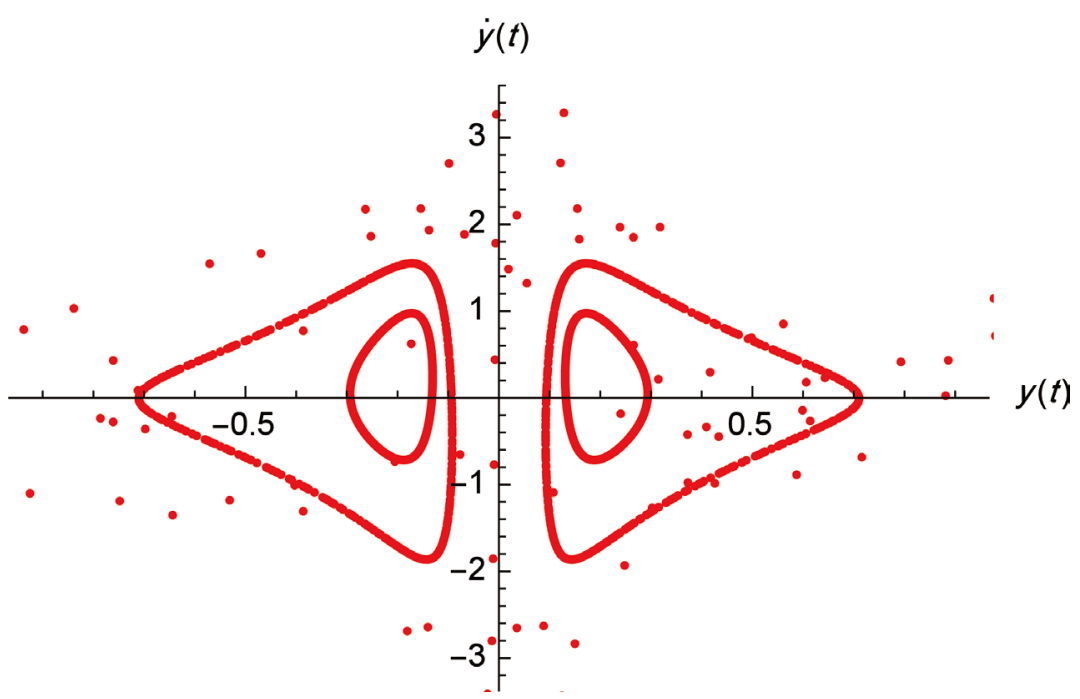

Figure 25. The invariant curves for $0<h \ll 1$ and $0<k \ll 1$ and $h=3 k$. 


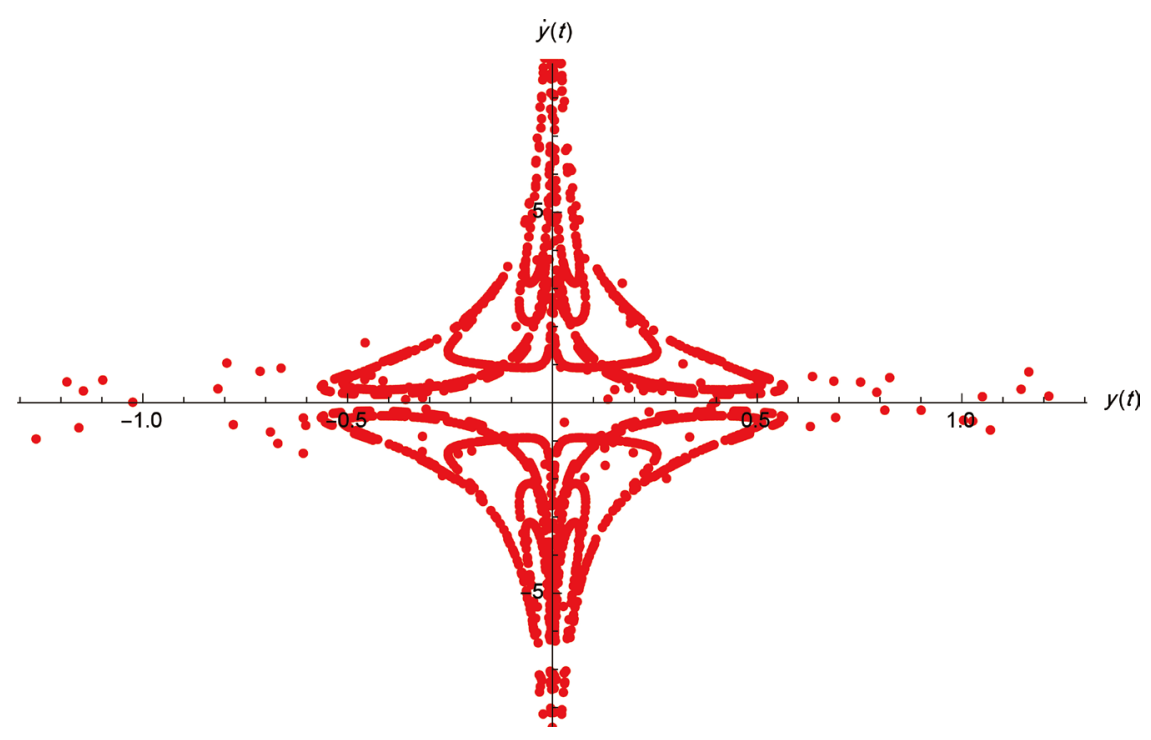

Figure 26. The invariant curves for $0<h \ll 1$ and $0<k \ll 1$ and $k=5000 h$.

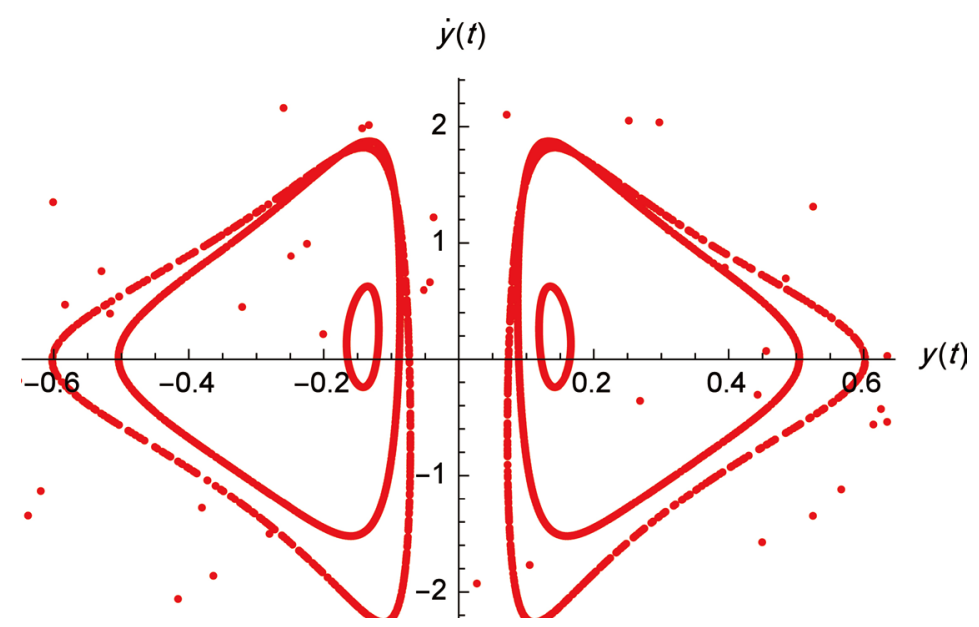

Figure 27. The invariant curves for $0<h \ll 1$ and $0<k \ll 1$ and $h=50 k$.

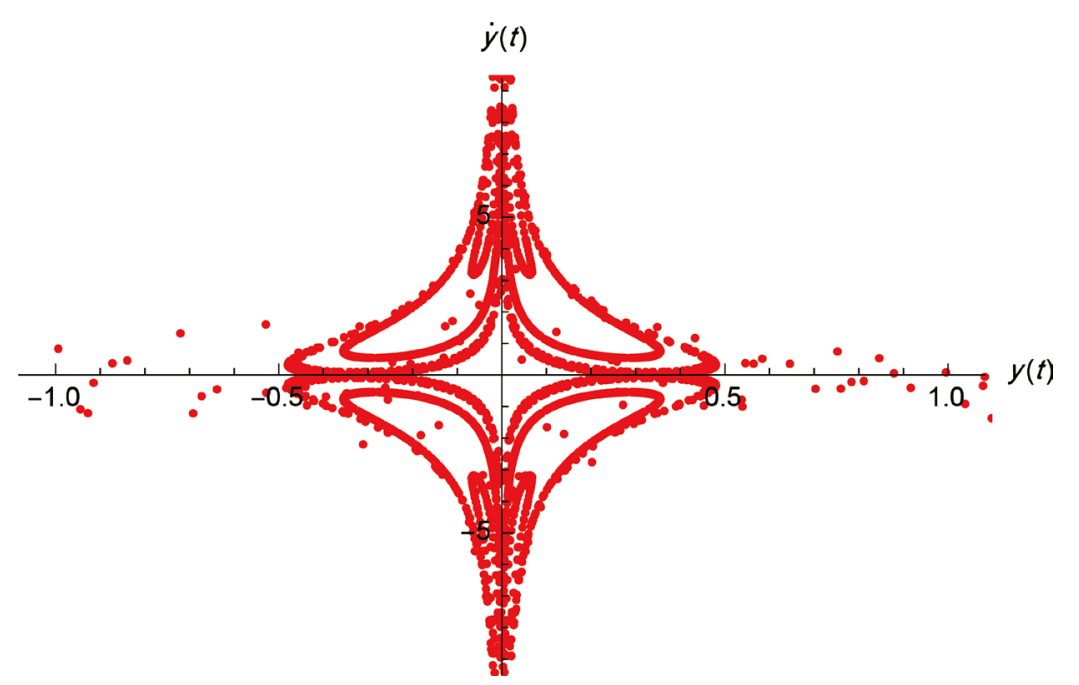

Figure 28. The invariant curves for $h \ll 0, k<0$ for small value of $\mathrm{h}$ and large value of $k$ such that $k=100$ times of $h$, the curves seem to be a closed curves. 


\section{Conclusions}

We conclude some results related to the behavior of the motion:

1) The elliptic points in the figures are stable in the Lyapunov sense, because a small disturbance will result in a closed trajectory that surrounds it and along which the state of the system remains close to these points.

2) The hyperbolic points are unstable because any small disturbance will result in a trajectory on which the state of the system deviates more and more from these points as $t$ goes to infinity.

\section{References}

[1] Leimanis, E. (1965) The General Problem of the Motion of Coupled Rigid Bodies about a Fixed Point. Springer-Verlag, Berlin-Heidelberg, New York. https://doi.org/10.1007/978-3-642-88412-2

[2] Kovalevskaya, S. (1889) Sur le probléme de la rotation d'un corps solide autour d'un point fixe. Acta Mathematica, 12, 77-232.

[3] Golubev, V.V. (1953) Lectures on Integration of the Equations of Motion of Rigid Body about a Fixed Point. GITTL, Moscow.

[4] Kozlov, V.V. (1983) Integrability and Non-Integrability in Hamiltonian Mechanics. Russian Mathematical Surveys, 38, 1-76.

[5] El-Sabaa, F. (1983) Solution of the Equations of the Problem of Motion of a Heavy Rigid Body about a Fixed Point in the Kovaleveskaya Case Using $\theta$-Function, Celestial Mechanics, 29, 249-253. https://doi.org/10.1007/BF01229138

[6] Poincaré, H. (1957) Les méthodes nouvelles de la mécanique céleste.

[7] Fomenko, A.T. (1988) Integrability and Nonintegrability in Geometry and Mechanics. Kluwer Academic Publishers, Dordrecht. https://doi.org/10.1007/978-94-009-3069-8

[8] Kozlov, V.V. (1976) Splitting of the Separatrices in the Perturbed Euler-Poinsot Problem. Moskovskii Universitet, Vestnik, Seriia I: Matematika, Mekhanika, 31, 99-104.

[9] Henon, M. and Heiles, C. (1964) The Applicability of the Third Integral of Motion: Some Numerical Experiments. The Astronomical Journal, 69, 73.

[10] Tabor, M. (1988) Chaos and Integrability in Nonlinear Dynamics. A Wiley-Interscience Publication, New York. 
Submit or recommend next manuscript to SCIRP and we will provide best service for you:

Accepting pre-submission inquiries through Email, Facebook, LinkedIn, Twitter, etc. A wide selection of journals (inclusive of 9 subjects, more than 200 journals)

Providing 24-hour high-quality service

User-friendly online submission system

Fair and swift peer-review system

Efficient typesetting and proofreading procedure

Display of the result of downloads and visits, as well as the number of cited articles Maximum dissemination of your research work

Submit your manuscript at: http://papersubmission.scirp.org/

Or contact jamp@scirp.org 